\title{
1/30 scale of Japan topography model realized with Minecraft
}

\author{
Yoshiharu Nishioka ${ }^{\mathrm{a}, *}$ \\ ${ }^{a}$ Geological Survey of Japan, AIST, y-nishioka@aist.go.jp \\ * Corresponding author
}

Keywords: Minecraft, Learning support, Topography, Elevation, JavaScript

\section{Abstract:}

The purpose of this research is to provide an environment for children to learn about Japan's topography. In recent years, games have been attracting attention as tools for active learning, which has been a departure from conventional knowledge acquisition bias education. I focused on 3D sandbox game called Minecraft (Mojang/Microsoft) which is popular among children. In Minecraft, players can freely move in the 3D world and create buildings. Minecraft can reproduce 3D topography and simulate it, so it has high potential as a geography and geology education.

I have created 1/30 scale of Japanese terrain model for Minecraft Java 1.12.2. The created data can be downloaded and used freely. The usage method is simple, it only copies it to the prescribed directory of the environment where Minecraft was installed.

For the elevation data necessary for model creation, I use the elevation tile released by the Geospatial Information Authority of Japan as open Data. This elevation tile covers the whole of Japan with 10m mesh accuracy. This elevation tile is an application of the tiled map image often used in Web maps to data representation. I call such a data set "Data Tile Map". Each pixel holds the elevation as a signed integer in cm units, using 24-bit RGB values.

The creation program is written in JavaScript and can be executed in an environment with a web browser. With the creation program you can change the output resolution (zoom level) and vertical direction exaggeration ratio. In the future, I plan to add functions to correspond to the terrain of the world and create data by specifying the area.

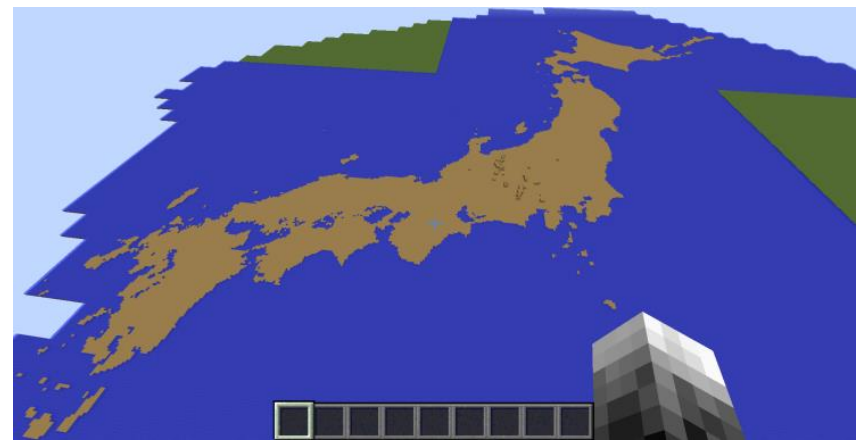

Figure 1. Japan of 1/4000 scale seen in Minecraft.

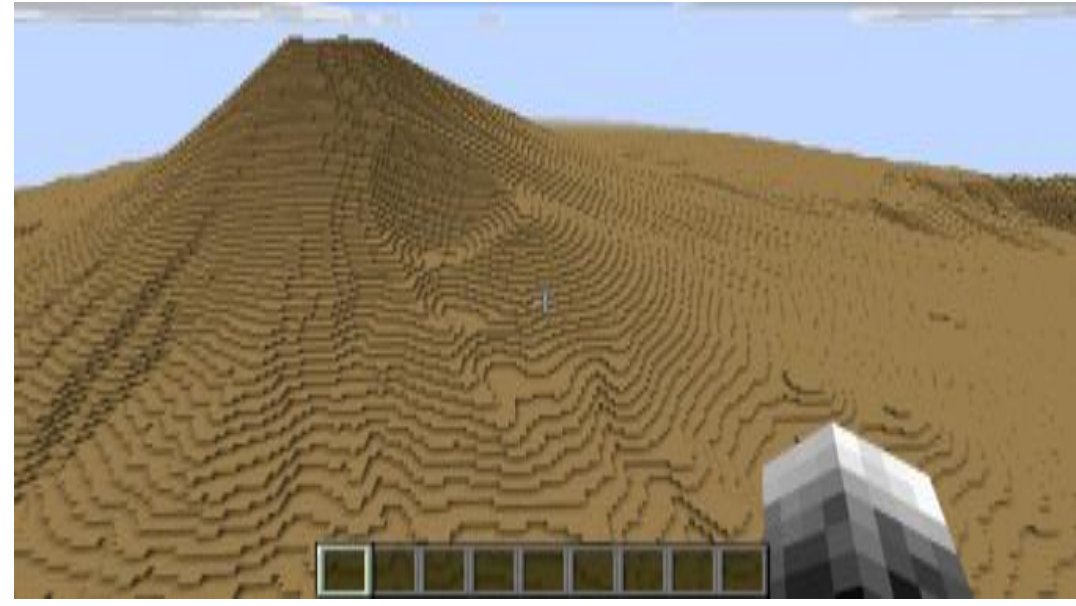

Figure 2. Mt. Fuji of 1/30 scale seen in Minecraft. 